\title{
Médiévales
}

Langues, Textes, Histoire

72 | printemps 2017

Roman du Genji et société aristocratique au Japon

\section{Elma BRENNER, Leprosy and Charity in Medieval Rouen}

Woodbridge, The Boydell Press, 2015 (« The Royal Historical Society Studies in History, New Series »)

\section{Bruno Tabuteau}

\section{OpenEdition}

Journals

Édition électronique

URL : https://journals.openedition.org/medievales/8142

DOI : $10.4000 /$ medievales.8142

ISSN : 1777-5892

Éditeur

Presses universitaires de Vincennes

Édition imprimée

Date de publication : 22 juin 2017

Pagination : 201-204

ISBN : 978-2-84292-612-0

ISSN : 0751-2708

\section{Référence électronique}

Bruno Tabuteau, «Elma brenner, Leprosy and Charity in Medieval Rouen», Médiévales [En ligne], 72 | printemps 2017, mis en ligne le 08 juillet 2017, consulté le 24 avril 2022. URL : http://

journals.openedition.org/medievales/8142; DOI : https://doi.org/10.4000/medievales.8142

Ce document a été généré automatiquement le 24 avril 2022.

Tous droits réservés 


\title{
Elma BRENNER, Leprosy and Charity in Medieval Rouen
}

\author{
Woodbridge, The Boydell Press, 2015 (« The Royal Historical Society \\ Studies in History, New Series »)
}

\section{Bruno Tabuteau}

\section{RÉFÉRENCE}

Elma BRENNER, Leprosy and Charity in Medieval Rouen, Woodbridge, The Boydell Press, 2015, XI-203 p. (« The Royal Historical Society Studies in History, New Series »)

En 2008, Elma Brenner avait soutenu avec succès sa thèse de doctorat à l'Ugniversité de Cambridge, sur la charité à Rouen aux $\mathrm{XII}^{\mathrm{e}}$ et $\mathrm{XIII}^{\mathrm{e}}$ siècles, à travers l'étude de l'importante maladrerie du Mont-aux-Malades. Elle en a tiré un livre qui étend le propos à l'ensemble des léproseries rouennaises et le prolonge chronologiquement jusqu'au bas Moyen Âge, en s'interrogeant sur les véritables effets socioculturels, à l'échelle d'une grande ville européenne, d'une maladie qui connote si funestement nos modernes représentations de cette époque. L'auteure a légitimement cherché à inscrire son travail dans le renouvellement historiographique qui, en deux à trois décennies, a profondément changé notre regard sur le sujet par une réévaluation critique de l'héritage stigmatisant $d u x x^{e}$ siècle. Dans cette perspective, elle a mesuré toute la portée de la question, qui s'est posée dans l'Église aux $\mathrm{XII}^{\mathrm{e}}$ et $\mathrm{XIII}^{\mathrm{e}}$ siècles, du statut religieux des lépreux vivant en communauté sur le modèle prégnant du monachisme, dans des léproseries dont le concile de Latran III (1179) a sanctionné l'autonomie ecclésiastique et qui n'ont pas manqué d'attirer la charité des fidèles inquiets d'un salut que pouvaient leur faire espérer les prières de pauvres malades éprouvés et non plus châtiés par Dieu et appelés ainsi eux-mêmes à la conversion. C'est du reste cette charité, au cœur de la doctrine chrétienne, qui a suscité la multiplication des fondations hospitalières aux $\mathrm{XII}^{\mathrm{e}}$ et $\mathrm{XIII}^{\mathrm{e}}$ siècles, quand l'essor économique, démographique et urbain de l'Occident en a accru les moyens matériels par 
l'enrichissement d'une élite sociale confrontée à l'ampleur d'une pauvreté que les lépreux incarnaient par excellence.

2 La métropole rouennaise, l'une des plus notables de «l'empire angevin », puis du royaume capétien, a possédé nombre de ces établissements. Au rang des léproseries, Elma Brenner consacre les deux chapitres initiaux de son livre au prieuré augustin du Mont-aux-Malades, sur lequel nous renseignent un gros fonds d'archives depuis le $\mathrm{XII}^{\mathrm{e}}$ siècle et d'autres sources remarquables telles que le registre des visites pastorales de l'archevêque Eudes Rigaud sous Saint Louis. Son histoire est caractéristique des maladreries médiévales. Ce prieuré est né dans la première moitié du XII ${ }^{e}$ siècle, sur une hauteur dominant la ville et la vallée de la Seine, de l'institutionnalisation (et non pas de la fondation actée) d'un possible groupe de malades préexistant, grâce au soutien des puissants laïcs et ecclésiastiques. Sa très ordinaire localisation dans la campagne périurbaine, sur la route de Dieppe, répondait aux exigences de la loi mosaïque, qui éloignait le lépreux "hors du camp", tout en profitant de l'implantation dans un espace productif, agricole et pastoral, de l'introduction dans les circuits commerciaux polarisés par la place de Rouen et de l'ouverture à l'aumône des passants sur un axe de circulation majeur. Une foire annuelle de huit jours s'y est tenue en outre par la faveur de Henri II Plantagenêt, qui a été un protecteur et bienfaiteur insigne de l'établissement après le meurtre de Thomas Becket dans sa cathédrale de Cantorbéry et sa précoce canonisation. Le primat d'Angleterre avait, de son vivant, bénéficié de l'amitié indéfectible du prieur dans sa fatale querelle avec le monarque. La pénitence royale a entraîné la refondation du prieuré Saint-Jacques par l'érection d'une nouvelle église sous le vocable du saint martyr. Au XIII ${ }^{\mathrm{e}}$ siècle, le Mont-aux-Malades a été de plus en plus impliqué dans la vie urbaine par l'augmentation significative de son patrimoine économique en ville, due à la charité des bourgeois et des habitants, qui a relayé celle de la royauté et de l'aristocratie anglo-normandes après 1204 et qui a pu être payée de retour par l'exclusivité de l'admission à la maladrerie des fidèles lépreux des vingt et une paroisses dites bientôt "du droit», soit deux paroisses de Rouen sur trois. La communauté du Mont a compté jusqu'à plus de soixante membres, religieux (dont des lépreux) et laïcs, séparant les hommes et les femmes et distinguant la moitié de malades des sains, chanoines et frères et sœurs lais : les « couvents » d'Eudes Rigaud, dont la distribution simple masquait une réalité compliquée par le poids des hiérarchies sociale, religieuse et institutionnelle extérieures auxquelles se rattachaient les individus (bourgeois, moines...).

3 À l'opposé du Mont s'élevait dans la vallée de la Seine "la Salle-aux-Puelles » (Aula Puellarum), qui fait principalement l'objet du troisième chapitre du livre d'E. Brenner. C'était un type de léproserie fort différent, unique en Normandie, car il s'agissait d'une communauté féminine à l'image de fondations anglaises, et peut-être aristocratique, installée par Henri II dans un manoir royal, dont témoigne aujourd'hui, aux voûtes du chœur de la chapelle, le beau programme pictural de l'Enfance du Christ exécuté sous son règne. Eudes Rigaud a fixé la discipline claustrale de ce prieuré de cinq à dix sœurs lépreuses qui, contrairement à ce que suppose l'auteure, n'observaient pas forcément la règle augustinienne ni n'étaient assistées d'une fraternité valide. Il n'est pas non plus nécessaire d'imputer à un diagnostic de lèpre erroné la présence d'une jeune noble saine cachée par les malades pour avoir forniqué avec le prêtre d'une autre léproserie de la vallée, liée à la Salle-aux-Puelles. En sus du Mont-aux-Malades et de la Salle-auxPuelles, des sources mentionnent huit petites maisons qui ceinturaient Rouen, dont 
celles dites précisément des " quatre portes », et qui, à l'instar du Mont, desservaient des paroisses de l'agglomération. Cependant, au XIII e siècle, l'une des huit se serait réduite à une éphémère cabane. Une neuvième n'a manifestement jamais existé.

Après avoir ainsi fait le tour des institutions charitables réservées aux lépreux, soulignant leur pleine insertion économique, sociale, juridique et culturelle dans l'aire urbaine et périurbaine rouennaise du second Moyen Âge, l'historienne traite dans les deux derniers chapitres de son livre de la lèpre sous l'angle médical, puis religieux, étant entendu qu'on jugeait alors la santé du corps tributaire de celle de l'âme. Au vrai, le dossier est à peu près vide sur les pratiques médicales et chirurgicales relativement aux lépreux et aux léproseries à Rouen. Il n'est permis d'invoquer, au mieux, que la banale phlébotomie des sœurs de la Salle-aux-Puelles et de rares examens de lépreux non certifiés avant le XIV ${ }^{e}$ siècle. E. Brenner trouve davantage matière à développer sur le régime alimentaire et le confort de vie des malades. Or, le régime «rigaldien » des sœurs de la Salle-aux-Puelles était celui de moniales et du Mont; il paraît avoir dépendu bien plus du statut religieux des membres de la communauté que de leur état de santé. Quant au vestiaire, Eudes Rigaud a pu admettre que des chemises et draps de lin irriteraient moins les chairs ulcéreuses des sœurs de la Salle-aux-Puelles. Mais, comme le note E. Brenner pour les capes de fourrure, ces chemises et draps n'auraientils pas été accordés en définitive à des femmes de condition? Le port d'un manteau uniforme rousset, enfin, signalait leur appartenance à une communauté religieuse.

Parler des lépreux d'un point de vue pathologique et médical, c'est naturellement poser le problème de la contagion, lequel a évolué d'un XII siècle, où la théorie humorale était déterminante, à un $\mathrm{XIV}^{\mathrm{e}}$ siècle, où la peste a cautionné l'idée d'une contamination humaine. Entre ces deux bornes chronologiques, la prudence est de mise : comment croire à une crainte de la contagion lépreuse dans les années trente et quarante du $\mathrm{XIII}^{\mathrm{e}}$ siècle, quand les règlements archiépiscopaux pour le Mont-aux-Malades et pour la Salle-aux-Puelles dénoncent la fréquentation habituelle des malades par des visiteurs sains, au préjudice de la discipline religieuse ? L'auteure semble hésiter, en revanche, lorsque des statuts synodaux interdisent aux lépreux l'accès aux agglomérations et spécialement aux tavernes, tout en rappelant aux fidèles le devoir de charité dans le cadre paroissial, partant celui des léproseries. Elle y voit une volonté de contrôler le mouvement des lépreux dans la société urbaine, par souci d'ordre et de santé publics, sans prendre assez en considération tous les aspects d'une législation qui s'est avérée vaine au demeurant, les lépreux ne cessant pas de venir mendier en ville jusqu'aux portes de la cathédrale.

6 Pour ce qui est de la cure des âmes, celle-ci requérait un personnel et un environnement appropriés: les textes nous renseignent sur l'encadrement ecclésiastique des communautés; avec les vestiges des églises ou chapelles, ils nous informent pareillement sur ces espaces cultuels au Mont-aux-Malades (autels, luminaire, reliques), de même que sur la liturgie et sur les sacrements au Mont et à la Salle-aux-Puelles (messes et offices, confession et communion). Les léproseries étaient de surcroit des lieux de sépulture et de commémoration des morts, tant pour leurs habitants que pour leurs bienfaiteurs, dont deux belles pierres tombales de la fin des $\mathrm{XIII}^{\mathrm{e}}$ et XIV ${ }^{\mathrm{e}}$ siècles ont été conservées dans l'église Saint-Thomas du Mont-aux-Malades. La diffusion de la doctrine du purgatoire, au xiII siècle, poussait à intensifier les suffrages aussi dans les maisons hospitalières, ce qui réclamait de leurs membres une discipline de vie religieuse garante d'une qualité spirituelle, notamment par le respect 
de la clôture monastique. D'où ce paradoxe, à nos yeux, de la libération des âmes par l'enfermement des corps, la souffrance ainsi assumée frayant la voie de la rédemption. Il était loisible à l'auteure de relever là une cause essentielle de méprise des historiens, qui ont traditionnellement mis la réclusion des lépreux sur le compte de la santé publique.

Elma Brenner conclut son livre en vantant l'intérêt de cette nouvelle étude de cas, indispensable à notre compréhension de la lèpre médiévale ! On retiendra sa réflexion sur une marginalité qui ne concernait en somme que le misérable lépreux errant, parmi la foule des vagabonds et mendiants de tout poil est-on tenté d'ajouter, et non pas le malade inclus dans l'institution de la léproserie, intégré dans la société chrétienne. L'ouvrage se complète d'appendices qui répertorient et analysent 116 actes très majoritairement des $\mathrm{XII}^{\mathrm{e}}$ et $\mathrm{XIII}^{\mathrm{e}}$ siècles, d'une bonne bibliographie et d'un utile index onomastique et thématique. Il comporte également une dizaine d'illustrations in texto, sur les églises ou chapelles du Mont-aux-Malades et de la Salle-aux-Puelles surtout, un tableau des petites léproseries de l'agglomération et une carte de localisation des léproseries autour de Rouen (avec une confusion pour l'une d'elles). Au total, il faut saluer la parution du livre d'Elma Brenner. Il atteste, après d'autres, de l'extrême fécondité d'un champ de recherche encore trop facilement négligé cependant, alors que l'histoire des lépreux éclaire à bien des égards les sociétés de ces siècles lointains. Et particulièrement sous le rapport de la charité, une valeur clé du monde chrétien, répétons-le, que l'auteure a eu le mérite de privilégier. 